\title{
Minimalinvasive Chirurgie Immer sinnvoll?
}

Marty Zdichavsky • Andreas Kirschniak • Jörg Glatzle • Tobias Meile • Markus Küper • Hannes Gögele • Gregor Blank • Jonas Hoffmann • Dörte Wichmann • Alfred Königsrainer

Schlüssellochchirurgie, Knopfloch- oder laparoskopische Chirurgie - die minimalinvasive Chirurgie hat viele Namen. Sie alle zielen auf das wesentliche Merkmal des Verfahrens ab: das Operieren durch kleine Zugänge statt durch zentimeterlange Schnitte. Das bietet Vorteile, vor allem für den Patienten. Kein Wunder, dass die minimalinvasive Chirurgie in einigen Bereichen die offene OP schon als „Goldstandard“" abgelöst hat. Trotzdem: Nicht immer eignet sich die modernere Variante. Und der Erfolg hängt erheblich von der Erfahrung des Operateurs ab. Unsere Autoren geben einen Überblick über die minimalinvasive Technik.

\section{Grundlagen der minimalinvasiven Chirurgie}

Anwendungsgebiete Die minimalinvasive Chirurgie hat sich bereits in unterschiedlichen Fachbereichen etabliert. Beispiele hierfür sind thorakoskopische, gynäkologische, orthopädische oder unfallchirurgische Eingriffe. Auch in der Allgemein- und Viszeralchirurgie ist das Verfahren immer häufiger und mittlerweile etabliert bei folgenden Operationen:

- Diagnostische Laparoskopie

$\triangleright$ Klärung unklarer Befunde

$\triangleright$ Staginguntersuchung bei Tumoren

- Adhäsiolyse

- Biopsieentnahmen bei Tumorerkrankungen

- Gallenblasenentfernung (Cholezystektomie)

- Blinddarmentfernung (Appendektomie)

- Darmteilentfernungen $\triangleright$ Dünndarmteilresektion

$\triangleright$ Ileozäkal-, Sigma- und Rektumresektion

$\triangleright$ (Hemi-)Kolektomie

$\triangleright$ Rektopexie

- Stomaanlage

- Eingriffe am Magen

$\triangleright$ Fundoplikation (Antirefluxoperation)

$\triangleright$ Gastrektomie

$\triangleright$ Gastroenterostomie

$\triangleright$ Übernähung eines Ulcus ventriculi

- Adipositaschirurgie

$\triangleright$ Gastric-banding (Magenband)

$\triangleright$ Gastric-sleeve-Resektion

$\triangleright$ Roux-Y-Bypass

- Splenektomie

- Adrenalektomie

- Hernienchirurgie

$\triangleright$ Leistenhernien (transabdominelle präperitoneale Patchplastik (TAPP), total extraperitoneale Patchplastik (TEP))

$\triangleright$ Narbenhernien (intraperitoneales onlay mesh (IPOM))

\section{Vorbereitungen \\ $\nabla$}

Spezialtraining nötig Um die handwerklichen Fertigkeiten und das Arbeiten über Sicht auf den Monitor zu erlernen, gibt es spezielle Trainingszentren. Das Absolvieren der Grund- und Aufbaukurse im hauseigenen Trainingszentrum ist an

Tab. 1

\begin{tabular}{l|l|l}
\hline \multicolumn{2}{l}{ Grundausstattung für die minimalinvasive Chirurgie } & \\
\hline Instrumente & Material & Geräte \\
\hline Veres-Kanüle & - Gasschlauch & Gasinsufflator \\
- Spül-Sauger & Saug-Spül-Schläuche & Spülapparat \\
- Trokare mit $\emptyset 6$ und $11 \mathrm{~mm}$ & - steriler Kamerabezug & Kamera \\
- $0^{\circ}$-Optik oder $30^{\circ}$-Optik & - Elektrokabel & - Monitor \\
- Hakenelektrode & - Lichtkabel & - Lichtquelle \\
- Pinzette & & Stromapparat bipolar/ \\
D Fasszange & & monopolar \\
- Schere & &
\end{tabular}



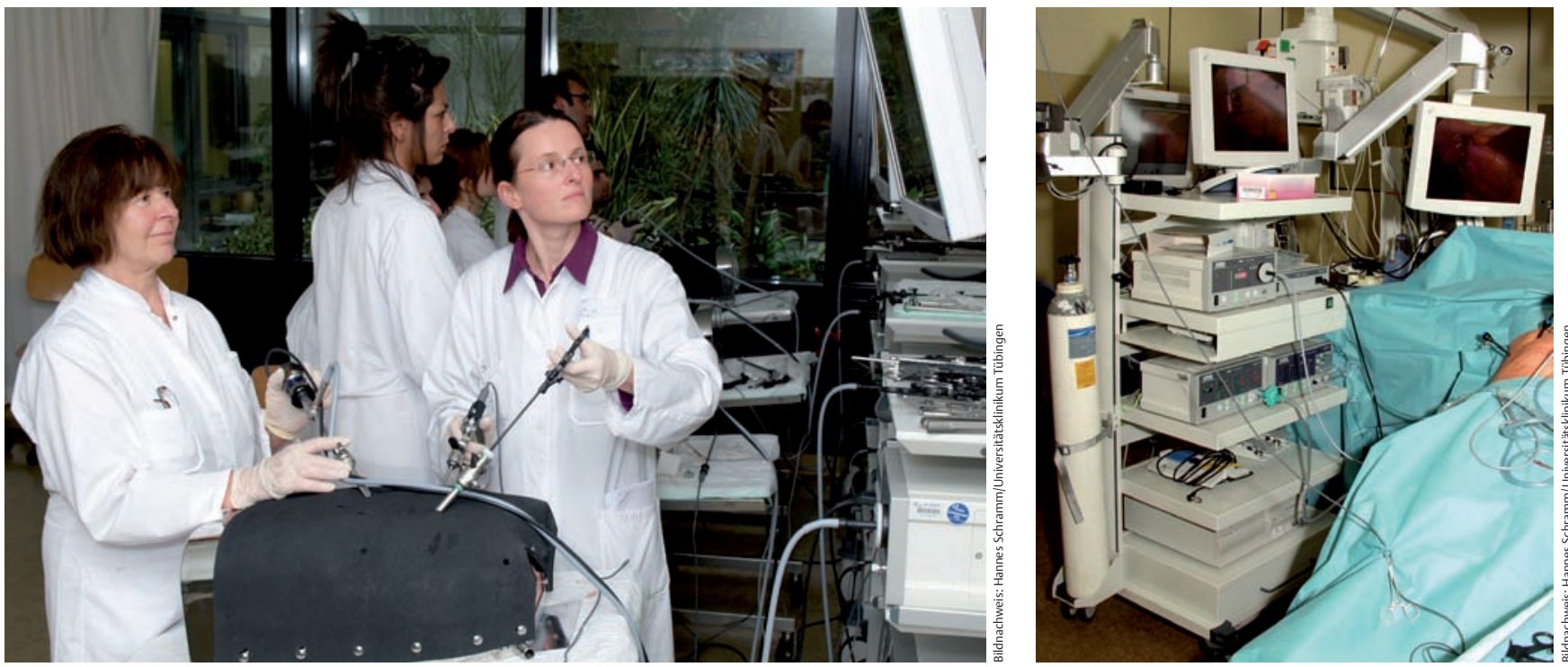

unserem Klinikum Pflicht. Am Trainer können Operationen an einem Phantom mit Tierorganen realitätsnah durchgeführt werden ( $\bullet$ Abb. 1 ). Dies dient einer optimalen Operationsvorbereitung.

Instrumentarium Für den minimalinvasiven Eingriff im Abdomen ist eine Grundausstattung notwendig. Sie umfasst das Instrumentarium, Geräte und zusätzliches Material ( $\bullet$ Tab. 1, Abb. 2).

\section{Die richtige Lagerung \\ $\nabla$}

Wichtig für den Zugang zu den Organen Bevor mit der eigentlichen Operation begonnen werden kann, muss der Patient korrekt gelagert werden. Die Lagerung ist besonders wichtig: Allein durch die Schwerkraft können Organe exponiert werden, ohne zusätzliche Haltevorrichtungen einbringen zu müssen.

Operationen im Oberbauch Bei Eingriffen an Galle, Magen oder Leber muss der Patient

- mit dem Oberkörper aufrecht gelagert werden. Hierzu sind Fußstützen notwendig (z. B. bei der Cholezystektomie).

- Bei der Fundoplikation sind die Arme in der Regel ausgelagert.

Operationen im Mittelbauch Die häufigsten Operationen im Mittelbauch betreffen Narbenhernien oder Darmeingriffe.

- Der Patient befindet sich in Rückenlagerung.

- Die Arme sind hierbei angelagert.

Operationen im Unterbauch Die Lagerung bei Leistenhernien, Appendektomie, Rektum- und Sigmaresektion sowie gynäkologischen Eingriffen unterscheidet sich von den bisher genannten.

- Es sollten Schulterstützen angebracht werden, da der Patient häufig in Kopftieflage gebracht werden muss.
- Für die Operationen am Rektum, Sigma und bei den gynäkologischen Eingriffen ist die Steinschnittlagerung obligat.

- Außerdem ist bei allen Operationen im Unterbauch ein Blasenkatheter sinnvoll, um eine gute Übersicht in den Douglas-Raum zu haben.

Besondere Lagerungen Bei Nieren- und Milzeingriffen ist die Seitenlagerung erforderlich:

- Bei der transabdominellen rechtsseitigen Nebennierenentfernung wird der Patient in die Linksseitenlage gebracht.

- Bei der linksseitigen transabdominellen Nebennierenentfernung und der Milzentfernung erfolgt die Lagerung in Rechtsseitenlage.

\section{Generelles Operationsprinzip \\ $\nabla$}

Einbringen des ersten Trokars Zunächst erzeugt man ein Pneumoperitoneum durch das Insufflieren von erwärmtem Kohlendioxid $\left(\mathrm{CO}_{2}\right)$.

- Der erste Zugang zur Bauchhöhle erfolgt durch einen offenen kleinen Bauchschnitt und das offene Einbringen des ersten Trokars.

- Alternativ kann man die Veres-Nadel (auch Veres-Kanüle genannt) blind einbringen und darüber das Pneumoperitoneum schaffen.

- Bei einem intraabdominellen Gasdruck von $15 \mathrm{mmHg}$ wird die Veres-Nadel wieder zurückgezogen und der erste Trokar entweder blind eingebracht oder bevorzugt ein Optiktrokar verwendet.

Vorteil des Optiktrokars (auch Sicherheitstrokar genannt): Er ist durchsichtig, und man kann mit der Kameraoptik unter Sicht - und nicht blind sämtliche Bauchwandschichten durchstoßen.

- Der Gasschlauch wird an den ersten Trokar angeschlossen, um so ein konstantes Pneumoperitoneum aufrecht zu erhalten. 
Setzen der weiteren Trokare Alle weiteren Trokare werden über kleine Hautschnitte jeweils unter Sicht durch die Bauchwand in das Abdomen eingebracht. Die Spitze der Trokare sollte hierbei stumpf sein, um Organverletzungen zu vermeiden. Die Optik und die Arbeitsinstrumente werden durch die Trokare in den Bauchraum eingeführt.

Nach der OP Nach Beendigung der Operation werden sämtliche Arbeitsinstrumente und die Trokare unter Sicht aus dem Abdomen entfernt.

- Ab einem Trokardurchmesser von $10 \mathrm{~mm}$ sollte ein Faszienverschluss erfolgen, um Trokarhernien zu vermeiden. Bei kleineren Trokaren ist dies nicht notwendig.

Prinzipiell gilt: Bei jeder Operation muss die Übersichtlichkeit gewahrt sein, um Begleitverletzungen zu vermeiden.

\section{Vor- und Nachteile der minimalinvasiven Technik \\ $\nabla$}

Vorteile für den Patienten Die Hauptvorteile der minimalinvasiven Chirurgie bestehen in der

- Minderung des Zugangstraumas zum Operationsgebiet und einem

- reduzierten Operationstrauma.

- Das Schmerzempfinden ist deutlich verringert, ebenso der Schmerzmittelverbrauch.

- Die Mobilisation und der Kostaufbau können in der Regel rasch erfolgen.

- Hierdurch vermindern sich Gesamtmorbidität und Liegezeit des Patienten.

- Die Rekonvaleszenzzeit ist im Gegensatz zu den offenen Operationen deutlich verringert, und

- der Patient wird schneller wieder arbeitsfähig.

Nachteile und Grenzen Die Grenzen und Risiken der minimalinvasiven Chirurgie liegen zum Teil in der Konstitution des Patienten.

- Bei kardialen oder pulmonalen Risiken kann es durch das Anlegen des Pneumoperitoneums zu Herzrhythmusstörungen oder Beatmungspro- blemen kommen. Das kann die Operation in dieser Technik unmöglich machen.

- Auch ein besonders adipöser intraabdomineller Situs, schwere entzündliche Befunde oder vorausgegangene offene Operationen können den Eingriff erheblich erschweren.

- In der Anfangsphase sind die Operationszeiten auch für Standardeingriffe in der Regel verlängert.

- Optimal ist ein eingespieltes Team von Narkosearzt, Operationsschwester, Assistenz und Operateur. Besonders die gute Teamarbeit zwischen dem Operateur und dem kameraführenden Assistenten ist umso wichtiger, je komplexer der Eingriff ist.

- Hauptgefahr ist die akzidentielle Organverletzung durch Stromweiterleitung oder Instrumente, die außerhalb des Gesichtsfelds liegen.

- Der Kostenaufwand ist auch für relativ einfache Eingriffe höher als bei der offenen Chirurgie.

Alle Vor- und Nachteile sind nochmals in $\bullet$ Tab. 2 zusammengefasst.

Nebenwirkungen Bei der minimalinvasiven Chirurgie können durch das Pneumoperitoneum unangenehme Begleiterscheinungen auftreten, wie

- Schulterschmerzen oder eine

- Reizung des Nervus phrenicus.

- In manchen Fällen kann eine Krepitation an der Haut festgestellt werden. Diese entsteht durch das Gas, das durch die Bauchwand entweicht.

\section{Praktische Beispiele}

\section{Laparoskopische Cholezystektomie}

Zum Standard avanciert Die minimalinvasive Entfernung der Gallenblase (Cholezystektomie, - Abb. 3) wird heutzutage in etwa $90 \%$ der Fälle laparoskopisch durchgeführt und gilt als der „Goldstandard“. Erich Mühe entfernte 1985 als Erster eine Gallenblase laparoskopisch.

Indikation Die häufigste Indikation sind symptomatische Gallensteine. Eingeklemmte Gallen-

\begin{tabular}{l|l} 
Vor- und Nachteile der minimalinvasiven Chirurgie auf einen Blick \\
\hline Vorteile & Nachteile \\
\hline Minderung des Zugangstraumas & Minderung des Gewebegefühls \\
- Minimierung des Präparationstraumas & längere Operationszeit in der Lernphase \\
- kosmetische Vorteile & Training und eingespieltes Team erforderlich \\
weniger Flüssigkeitsverlust & - ungere Materialkosten \\
- Minderingere Körperauskühlung & che Veränderungen \\
- reduzierter Schmerzmittelverbrauch & Einschränkungen bei voroperierten Patienten \\
raschere Genesung & problematisch bei kardiopulmonalen Erkran- \\
- kürzere Arbeitsunfähigkeit & kungen
\end{tabular}


steine im Ductus cysticus können akute kolikartige Schmerzen verursachen. Weiterhin können diese einen Gallenblasenhydrops mit einem Aufstau der Galle und folgend eine Entzündung der Gallenblase (Cholezystitis) hervorrufen.

Die laparoskopische Cholezystektomie wird zumeist elektiv durchgeführt. Die Gallenblasenwand ist zart und nicht entzündet. In aller Regel sind die anatomischen Strukturen gut zu erkennen.

Besonderheit Cholezystitis Eine akute Cholezystitis bei einem Steinleiden behandelt man zunächst antibiotisch und führt den Patienten frühzeitig der Cholezystektomie zu. Die Operation kann auch elektiv nach 6 Wochen im entzündungsfreien Intervall durchgeführt werden. Sogar bei akuter Cholezystitis ist eine minimalinvasive Cholezystektomie möglich. Diese Eingriffe sind jedoch anspruchsvoller und häufiger mit Komplikationen verbunden. Sie sollten daher geübten laparoskopischen Chirurgen vorbehalten bleiben.

Leitstrukturen der Gallenblase bei Cholezystektomie Gute Kenntnisse der Anatomie von

- Gallenblase,

- Ductus cysticus,

- Arteria cystica und

- hepatobiliärem (Calot'schem) Dreieck

sind unerlässlich, um Verletzungen zu vermeiden. Hierbei sind besonders die Normvarianten der A. cystica und des Ductus cysticus hervorzuheben.

Calot'sches Dreieck Das Calot'sche Dreieck wird nach medial vom Ductus hepaticus communis, nach lateral vom Ductus cysticus und nach kranial vom Leberunterrand gebildet. Die Arteria cystica verläuft normalerweise innerhalb des Calot'schen Dreiecks, wobei sie hier schräg einzieht und in 40-81\% der Fälle zwischen den Peritonealblättern etwas hinter dem Ductus cysticus gefunden wird $[1,2](\bullet$ Abb. 4$)$.

Arteria cystica Die Arteria cystica entstammt überwiegend der rechten A. hepatica. Die A. cystica verläuft durch das Calot'sche Dreieck und verzweigt sich unmittelbar vor der Einmündung in die Gallenblase in 2 aufwärts ziehende Äste.

- Der vordere Ast verläuft an der linken Seite der Gallenblase, während

- der hintere zwischen der dorsalen Gallenblase und der Leberloge verläuft.

Variationen der A. cystica finden sich in etwa 50\% aller Patienten [1]. Neben dem normalen Verlauf tritt auch eine vordere, hintere, mehrfache oder kurze A. cystica auf [3].

Ductus cysticus Der Ductus cysticus vereint sich mit dem Ductus hepaticus communis zum Ductus choledochus, der im Ligamentum hepatoduodenale verläuft und in den Zwölffingerdarm

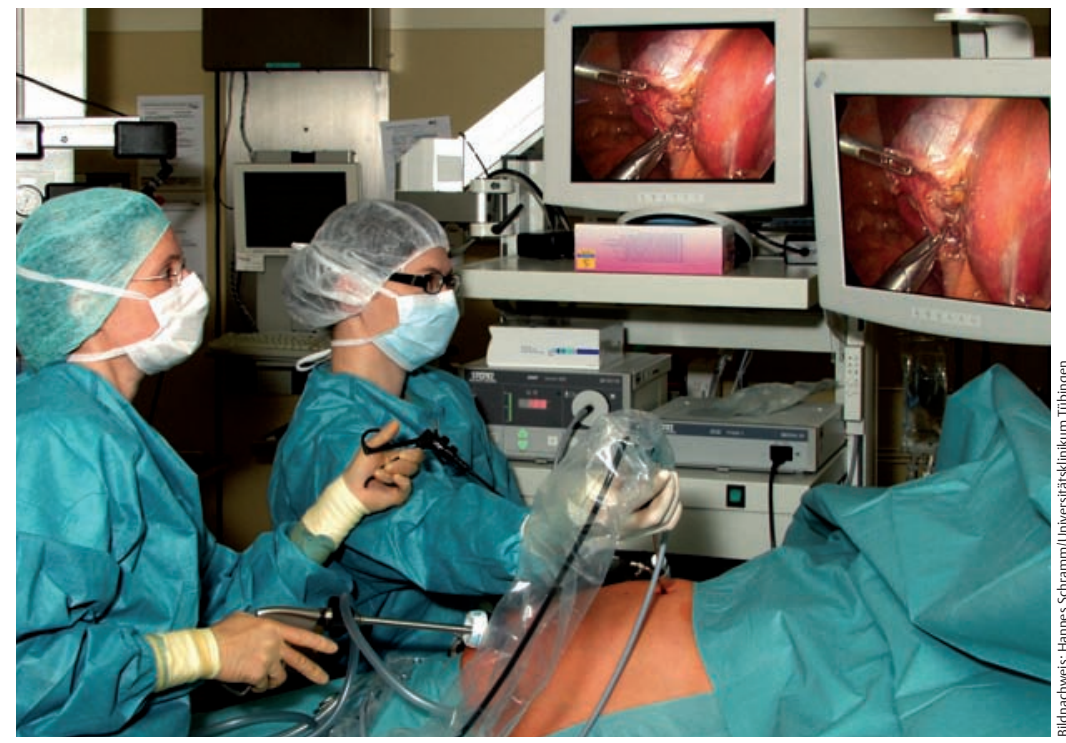

Abb. 3 Vom Pelvitrainer an

mündet. Der Verlauf des Ductus cysticus ist, wie die Anatomie der A. cystica, sehr variabel [4].

Es gibt auch akzessorische oder doppelte Gallengänge, die bei einer Cholezystektomie ebenfalls versorgt werden müssen.

den Patienten: Während Marty Zdichavsky (links) operiert, führt Assistenzärztin Jessica Lange bei der laparoskopischen Cholezystektomie die Kamera.
Zugangswege Über einen $10 \mathrm{~mm}$ langen Hautschnitt innerhalb des Bauchnabels wird für den 10-mm-Trokar der erste Zugang zur Bauchhöhle geschaffen. Über diesen Trokar kann man die $30^{\circ}$-Optik in das Abdomen einbringen. Anschließend wird unter Sicht ein weiterer 10-mm-Trokar im Epigastrium platziert. Er dient als Arbeitstrokar sowohl für 5-mm- als auch für 10-mm-Instrumente. Zwei weitere 5-mm-Arbeitstrokare werden rechts-lateral unterhalb des Rippenbogens gesetzt.

Operationsprinzip Der Patient befindet sich in Rückenlagerung mit ausgelagerten Armen und Fußstützen.

1. Nach dem Setzen des ersten Trokars wird der Operationstisch um $30^{\circ}$ aufgerichtet und zur linken Seite gedreht. Durch die Schwerkraft fallen die Darmschlingen nach kaudal, und die Gallenblase kann optimal exponiert werden.

2. Über den rechts-lateralen Trokar wird eine Fasszange eingeführt, die den Fundus der Gallenblase greift und nach kranial über den Leberrand zieht. Damit wird die Leber automatisch nach oben gehalten.

Prinzipiell erfolgt die gesamte Präparation so gallenblasennah wie möglich, um Verletzungen des Ductus hepatocholedochus zu vermeiden.

3. Das Peritoneum wird am Gallenblasenunterrand im Bereich des Calot'schen Dreiecks eröffnet. 
Abb. 4, links Leitstrukturen der Gallenblase: Kurz unterhalb des Leberrandes vereinigen sich die beiden Hauptgallengänge zum Ductus hepaticus communis. Ca. $3-4 \mathrm{~cm}$ distal davon mündet die Gallenblase über den Ductus cysticus ein. Diese Strukturen bilden das CalotDreieck. Danach beginnt der Ductus choledochus. Die arterielle Versorgung erfolgt über die Arteria cystica, die meist der A. hepatica dextra entspringt. Sowohl die Einmündung des Ductus cysticus als auch der Ursprung der A. cystica kann variieren!

Abb. 5, rechts Laparoskopische Cholezystektomie: Clip des Ductus cysticus (Pfeile), Darstellung der A. cystica (*).
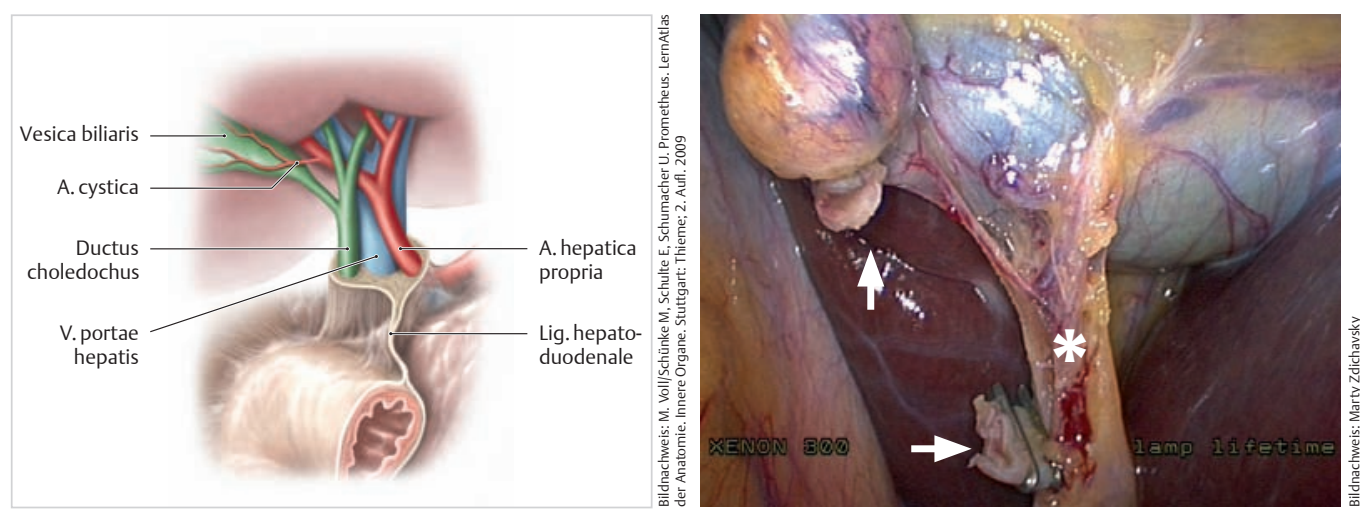

4. Es werden die A. cystica und der Ductus cysticus aufgesucht. Der Übergang des Infundibulums der Gallenblase in den Ductus cysticus muss unbedingt eindeutig dargestellt werden, um Verwechslungen mit dem Ductus choledochus zu vermeiden. Der eindeutige Übertritt der A. cystica zur Gallenblase ist obligat, um Verletzungen der A. hepatica dextra zu vermeiden.

Erst nach eindeutiger Darstellung der Strukturen (๑ Abb. 5) dürfen diese geclippt und durchtrennt werden. Üblicherweise werden dazu Titanclips verwendet.

5. Die Gallenblase wird mittels Elektrokoagulation aus dem Leberbett präpariert und im Bergebeutel über einen der 10-mm-Trokare geborgen. Der Trokar wird zuvor entfernt und die Faszie gespreizt.

6. Schließlich wird das Operationsgebiet nochmals inspiziert, bevor die Trokare unter Sicht aus dem Abdomen entfernt werden. Bei den 10-mm-Zugängen erfolgt ein Faszienverschluss. Das Einbringen einer Zieldrainage ist bei einem Gallenblasenempyem oder in Fällen bei akuter oder gangränöser Cholezystitis empfehlenswert.

Risiken und Komplikationen Selbst bei sorgfältigster Präparation kann es auch bei diesem Routineeingriff zu Komplikationen kommen.

- Eine gefürchtete Komplikation ist die Verletzung des Ductus hepatocholedochus.

In den Anfangszeiten der Laparoskopie war die Verletzungsrate höher als bei der offenen Cholezystektomie. In den vergangenen Jahren ging sie jedoch immer weiter zurück und liegt seit dem Jahr 2006 niedriger $(0,1 \%)$ als bei der offenen Technik (0,2-0,3\%) [5-7].

\section{Laparoskopische Appendektomie}

Vorteile überwiegen Die Appendektomie wird gewöhnlich bei einer akuten Entzündung des Wurmfortsatzes (Appendizitis) durchgeführt. Die weltweit erste laparoskopische Appendektomie erfolgte 1980 durch den Gynäkologen Kurt Semm an der Christian-Albrechts-Universität zu Kiel [8, 9].

- Bei einer unkomplizierten Appendizitis bietet der minimalinvasive Zugang Vorteile gegenüber der offenen Operation [10-18]. Kirshtein et al. [19] zeigten auch für die komplizierte Appendizitis keine erhöhte Komplikationsrate.

- Neben der kosmetischen Überlegenheit kann bei der Laparoskopie zudem das gesamte Abdomen exploriert werden, einschließlich der weiblichen inneren Geschlechtsorgane.

- Die postoperative Morbidität ist deutlich geringer als bei der Laparotomie [20, 21]. häufigste Indikation zur Appendektomie dar. Aber auch rezidivierende rechtsseitige Unterbauchschmerzen können nach Ausschluss anderer Erkrankungen (z.B. Salpingitis, Zystitis, chronisch entzündliche Darmerkrankungen) zur Appendektomie führen. In 10-20\% aller Appendektomien lässt sich histologisch eine neurogene Appendizitis nachweisen [22, 23]. Daher sollte bei gegebener Indikationsstellung zur Laparoskopie auch eine makroskopisch unauffällige Appendix entfernt werden.

Zugangswege Der Zugang für die Optik liegt gewöhnlich im Nabel oder knapp darunter. Es wird ein $10 \mathrm{~mm}$ langer Hautschnitt für einen Trokar und eine $30^{\circ}$-Optik (beide $10 \mathrm{~mm}$ ) angelegt. Ein 5-mm-Trokar im mittleren Unterbauch knapp oberhalb der Schambehaarung sowie ein weiterer 5- oder 10-mm-Trokar im linken Unterbauch dienen als Arbeitszugänge.

Operationsprinzip Der Patient befindet sich in Rückenlagerung mit angelagerten Armen und Schulterstützen. Die Appendix kann jede denkbare Lage einnehmen, besonders die retrozäkal
Indikation Die akute Appendizitis stellt die 



Abb. 6, links Laparoskopischer Befund: phlegmonöse Appendizitis.

Abb. 7, rechts Unterbinden der Appendixbasis mit zwei Röderschlingen.

gelegene Appendix ist in manchen Fällen schwierig aufzufinden. Dann hilft es, den Patienten etwas kopftief zu lagern und den Operationstisch nach links zu drehen.

1. Die Mesoappendix und die darin verlaufende A. appendicularis werden entweder mittels Clips oder Elektrokoagulation durchtrennt. Hierbei ist die bipolare Elektrokoagulation empfehlenswert, um ungewollte thermische Schäden an naheliegenden Darmschlingen zu vermeiden.

2. Die Appendixbasis muss eindeutig dargestellt werden und kann basisnah mit zwei Röderschlingen unterbunden werden ( $\bullet$ Abb. 6,7$)$. Um Stuhlaustritt aus der Appendix zu vermeiden, kann man eine dritte Röderschlinge oberhalb der vorgesehenen Absetzungsebene platzieren.

Erst nach eindeutiger Darstellung und sicherer Versorgung der Appendixbasis wird der Eingriff fortgesetzt.

3. Die Appendix wird durchtrennt und im Bergebeutel geborgen. Reicht die Entzündung bis an die Appendixbasis heran, kann diese (oder sogar ein Teil des Zäkums) mittels Stapler durchtrennt werden.

4. Bei hochgradig entzündlichen Befunden empfiehlt sich die Anlage eine Zieldrainage.

Aufgepasst! Besondere Aufmerksamkeit verdient die Mukozele der Appendix: Sie kommt mit einer Prävalenz von 0,2-0,3\% aller Appendektomien vor [24]. Es gibt 4 Ausprägungen der Appendixmukozele:

- Retentionszyste

- Mukosahyperplasie

- muzinöses Zystadenom

- muzinöses Zystadenokarzinom [25]
Cave Die intraoperative Perforation von Appendixmukozelen ist unbedingt zu vermeiden: Perforierte Mukozelen können sich zu einem Pseudomyxoma peritonei entwickeln und eine Peritonektomie mit hyperthermer intraperitonealer Chemotherapie (HIPEC) nach sich ziehen.

Weitere Risiken und Komplikationen Es können Verletzungen am Darm oder Harnleiter vorkommen.

- In etwa 20-30\% der Fälle einer akuten Appendizitis besteht bereits eine Perforation (sog. komplizierte Appendizitis) [26].

- Es werden immer wieder erhöhte Abszessraten nach laparoskopischer Appendektomie bei komplizierter Appendizitis beschrieben [27, 28].

$\triangleright$ Die Benutzung eines Klammernahtapparates zum Absetzen der Appendixbasis minimiert diese Gefahr.

$\triangleright$ Abszesse können auch entstehen, wenn während des Eingriffs weiträumig gespült und dadurch die Umgebung kontaminiert wurde. Allerdings deckt sich die Beobachtung von Katsuno et al. [21] mit unserer Erfahrung, dass das Auftreten von intraabdominellen Abszessen nach laparoskopischer Appendektomie mit zunehmender Expertise der Operateure abnimmt.

- Ein Nachteil der minimalinvasiven Appendektomie ist, dass Ungeübte den Eingriff nicht einfach erweitern können, falls z.B. wegen des intraoperativen Befundes eine Ileozäkalresektion notwendig wird.

Fazit Die minimalinvasive Chirurgie ist für verschiedene Operationen heute der „Goldstandard“. Lange Operationszeiten und erhöhte Komplikationsraten wurden mit zunehmender Erfahrung minimiert. Hervorzuheben sind die kosmetischen Vorteile, geringe Gesamtmorbidität und rasche Rekonvaleszenz. 


\section{Kernaussagen}

- Für das minimalinvasive Operieren ist ein spezielles Training in Trainingszentren erforderlich.

- Ein eingespieltes Operationsteam ist hilfreich.

- Das Verfahren bietet Vorteile für Gesamtmorbidität, Rekonvaleszenz und Kosmetik. Die Patienten sind früh wieder körperlich belastbar.

- Rezidivierende laparoskopische Eingriffe sind möglich, da wenig Verwachsungen auftreten.

- Hohe Materialkosten sind ein wesentlicher Nachteil der minimalinvasiven Chirurgie.

- Bei unübersichtlichen Verhältnissen sollte rechtzeitig die Konversion zur offenen OP erfolgen. Falscher Ehrgeiz ist lebensgefährlich für den Patienten!

- Bei der Cholezystektomie gilt grundsätzlich: Die Präparation erfolgt gallenblasennah und die Anatomie muss stets klar dargestellt sein, bevor eine Struktur geclippt oder durchtrennt werden darf.

- Bei der Appendektomie muss die Appendixbasis eindeutig dargestellt und versorgt sein, bevor die Appendix durchtrennt wird. Bei eventuell vorhandener Mukozele ist deren Perforation unbedingt zu vermeiden.

\section{Literaturverzeichnis}

1 Balija M, Huis M, Stulhofer M, Nikolic V. Contribution to the nomenclature of variations of the cystic artery. Chirurg 2001; 72: 154-158

2 Suzuki M, Akaishi S, Rikiyama T, Naitoh T, Rahman MM, Matsuno S. Laparoscopic cholecystectomy, Calot's triangle, and variations in cystic arterial supply. Surg Endosc 2000; 14: 141-144

\section{Literatur online}

Das vollständige Literaturverzeichnis zu diesem Beitrag finden Sie im Internet:

Abonnenten und Nicht-
abonnenten können
unter „www.thieme-
connect.de/ejournals“ die
Seite der Lege artis aufru-
fen und beim jeweiligen
Artikel auf „Ergänzendes
Material“ klicken - hier ist
die Literatur für alle frei
zugänglich.
Abonnenten können alter-
nativ über ihren persön-
lichen Zugang an das Lite-
raturverzeichnis gelan-
gen. Wie das funktioniert,
lesen Sie unter: http://
www.thieme-connect.de/
ejournals/
help\#SoRegistrieren
ria cystica. Anat Embryol 1926; 81: 601-617

4 Lamah M, Karanjia ND, Dickson GH. Anatomical variations of the extrahepatic biliary tree: review of the world literature. Clin Anat 2001; 14: 167-172.

5 BQS 2006, Bundesgeschäftsstelle Qualitätssicherung gGmbH, Qualitätsindikator: Eingriffsspezifische Komplikationen (Cholezystektomie).

6 BQS 2007, Bundesgeschäftsstelle Qualitätssicherung gGmbH, Qualitätsindikator: Eingriffsspezifische Komplikationen (Cholezystektomie).

7 BQS 2008, Bundesgeschäftsstelle Qualitätssicherung gGmbH, Qualitätsindikator: Eingriffsspezifische Komplikationen (Cholezystektomie).

8 Semm K. Endoscopic appendectomy. Endoscopy 1983; 15: 59-64

9 Semm K. Technical surgical steps of endoscopic appendectomy. Langenbecks Arch Chir 1991; 376: 121-126

10 Chung RS, Rowland DY, Li P, Diaz J. A meta-analysis of randomized controlled trials of laparoscopic versus conventional appendectomy. Am J Surg 1999; 177: 250-256

11 Garbutt JM, Soper NJ, Shannon WD, Botero A, Littenberg B. Meta-analysis of randomized controlled trials comparing laparoscopic and open appendectomy. Surg Laparosc Endosc 1999; 9: 17-26

12 Golub R, Siddiqui F, Pohl D. Laparoscopic versus open appendectomy: a metaanalysis. J Am Coll Surg 1998; 186:

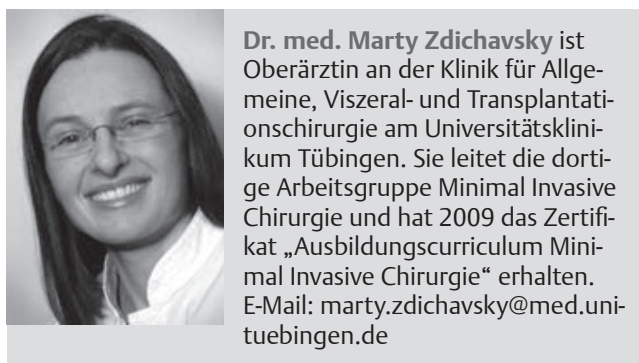

Dr. med. Andreas Kirschniak ist Facharzt an der Klinik für Allgemeine, Viszeral- und Transplantationschirurgie am Universitätsklinikum Tübingen.

E-Mail: andreas.kirschniak@med.uni-tuebingen.de

PD Dr. med. Jörg Glatzle ist Oberarzt an der Klinik für Allgemeine, Viszeral- und Transplantationschirurgie am Universitätsklinikum Tübingen.

E-Mail: joerg.glatzle@med.uni-tuebingen.de

Dr. med. Tobias Meile ist Facharzt an der Klinik für Allgemeine, Viszeral- und Transplantationschirurgie am Universitätsklinikum Tübingen.

E-mail: tobias.meile@med.uni-tuebingen.de

Dr. med. Markus Küper ist Assistenzarzt an der Klinik für Allgemeine, Viszeral- und Transplantationschirurgie am Universitätsklinikum Tübingen.

E-Mail: markus.kueper@med.uni-tuebingen.de

Dr. med. Hannes Gögele ist Assistenzarzt an der Klinik für Allgemeine, Viszeral- und Transplantationschirurgie am Universitätsklinikum Tübingen.

E-Mail: hannes.goegele@med.uni-tuebingen.de

Dr. med. Gregor Blank ist Assistenzarzt an der Klinik für Allgemeine, Viszeral- und Transplantationschirurgie am Universitätsklinikum Tübingen.

E-Mail: gregor.blank@med.uni-tuebingen.de

Dr. med. Jonas Hoffmann ist Assistenzarzt an der Klinik für Allgemeine, Viszeral- und Transplantationschirurgie am Universitätsklinikum Tübingen. E-Mail: jonas.hoffmann@med.uni-tuebingen.de 545-553
Dr. med. Dörte Wichmann ist Assistenzärztin an der Klinik für Allgemeine, Viszeral- und Transplantationschirurgie am Universitätsklinikum Tübingen.

E-Mail: doerte.wichmann@med.uni-tuebingen.de

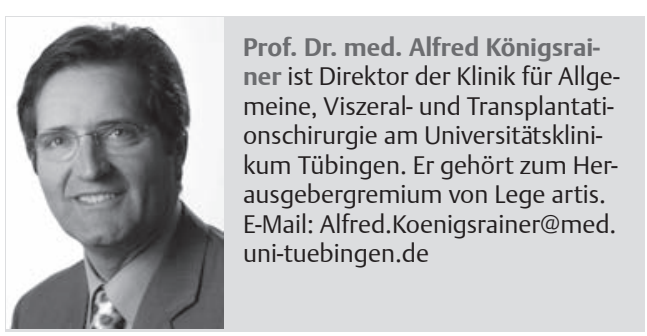

\section{Interessenkonflikt}

Die Autoren erklären, dass keine wirtschaftlichen oder persönlichen Verbindungen bestehen.

Beitrag online zu finden unter http://dx.doi. org/10.1055/s-0031-1272353 